\title{
Girls' preferences for HPV vaccination: a discrete choice experiment
}

Esther W. de Bekker-Grob ${ }^{\mathrm{a},{ }^{*}}$, Robine Hofman ${ }^{\mathrm{a}}$, Bas Donkers ${ }^{\mathrm{b}}$, Marjolein van Ballegooijen ${ }^{\mathrm{a}}$, Theo J.M. Helmerhorst ${ }^{\mathrm{c}, \mathrm{d}}$, Hein Raat ${ }^{\mathrm{a}}$, Ida J. Korfage ${ }^{\mathrm{a}}$

The Netherlands

${ }^{\text {a }}$ Dept of Public Health, Erasmus MC - University Medical Centre Rotterdam

${ }^{\mathrm{b}}$ Dept of Business Economics, Erasmus University, Rotterdam

${ }^{\mathrm{c}}$ Dept of Obstetrics and Gynaecology, Erasmus MC - University Medical Centre Rotterdam

${ }^{\mathrm{d}}$ Dutch Association of Obstetrics and Gynaecology, Utrecht

\section{* Corresponding author}

Esther W. de Bekker-Grob, PhD

Dept. of Public Health, Erasmus MC - University Medical Centre Rotterdam

PO Box 2040, 3000 CA Rotterdam, The Netherlands

Tel: $\quad+31-10-7043954$

Fax: $\quad+31-10-7044724$

email: e.debekker@erasmusmc.nl

Word account abstract: 156 ; Word account manuscript: 3,853

Table count: 5 ; Figure count: 2

Funding sources: grant support was from the Dutch Cancer Society (no. EMCR 2008-3992).

The authors declare their independence from the funder. 


\begin{abstract}
A discrete choice experiment was developed to investigate if girls aged 12-16 years make trade-offs between various aspects of human papillomavirus (HPV) vaccination, and to elicit the relative weight that girls' place on these characteristics. Degree of protection against cervical cancer, protection duration, risk of side-effects, and age of vaccination, all proved to influence girls' preferences for HPV vaccination. We found that girls were willing to trade-off $38 \%$ protection against cervical cancer to obtain a life-time protection instead of a protection duration of 6 years, or $17 \%$ to obtain an HPV vaccination with a 1 per 750,000 instead of 1 per 150,000 risk of serious side-effects. We conclude that girls indeed made a trade-off between degree of protection and other vaccine characteristics, and that uptake of HPV vaccination may change considerably if girls are supplied with new evidence-based information about the degree of protection against cervical cancer, the protection duration, and the risk of serious side-effects.
\end{abstract}

Keywords: Cervical cancer; Human papillomavirus; Vaccination; Preferences; Discrete choice experiment

Abbreviated title: Girls’ preferences for HPV vaccination 


\section{Introduction}

In countries with cytological screening programmes the mortality of cervical cancer has significantly decreased [1]. Since the discovery of human papillomavirus (HPV) as the cause of cervical cancer [2], new types of cervical cancer prevention, such as HPV screening and HPV vaccination, have been developed [3]. Currently HPV vaccines are available against HPVs 16 en 18, which have been estimated to cause $73-76 \%$ of cases of cervical cancer in Europe $[4,5]$. HPV vaccination is useful for women who have not been previously infected with these HPV types since the protection against cancer for women with existing or previous infections of type 16 or 18 is low. By the end of 2008 fifteen countries of the European Union had decided to introduce HPV vaccination into their national immunisation schedule for adolescent girls, while another six have started the decision-making process with a recommendation favouring introduction [6].

Attitude towards and uptake of the offered HPV vaccine may be influenced by its perceived advantages and drawbacks. Individuals may be willing to undergo an HPV vaccination despite several drawbacks (risk of side effects, injections needed) in order to maximize health benefit or, vice versa, they may accept a lower health benefit in order to avoid side-effects of vaccination. Research has shown that preferences (i.e. individual's valuation) can have a major impact on the willingness to use health care services [7]. Several qualitative studies gave some insights into girls' preferences for HPV vaccination [8-10]. However, quantitative studies investigating girls' preferences for HPV vaccination and their willingness to trade-off between protection against cervical cancer and other characteristics of HPV vaccination are lacking.

Therefore, this study investigates the preferences of girls aged 12-16 years for HPV vaccination through a discrete choice experiment (DCE), a quantitative approach that is increasingly used in health care $[11,12]$. 


\section{Materials and Methods}

\subsection{HPV vaccination}

In the Netherlands, a National Immunisation Programme (NIP) provides vaccinations against diphtheria, pertussis (whooping cough), tetanus, polio, type B Haemophilus influenzae, hepatitis B, mumps, measles, rubella (German measles) and meningococcosis C. In 2009, HPV vaccinations for 12-year-old girls (given as a series of three injections) were added to the NIP. To begin with, a catch-up programme was organised for girls aged 13 to 16 years. In the Netherlands, the HPV vaccine Cervarix is used, which protects against HPV-16 and HPV18. Parental consent for this vaccination is not needed as teenagers at the age of 12 years or older are officially allowed to decide for themselves whether they want to be vaccinated. All Dutch NIP vaccinations are offered free of charge.

\subsection{Discrete Choice Experiment}

DCEs, with their origin in marketing, are a novel approach to assess preferences for medical interventions. In DCEs it is assumed that a medical intervention, such as a vaccination programme, can be described by its characteristics (attributes; e.g. protection duration).[13] Those characteristics are further specified by variants of that characteristic (attribute levels; e.g. for protection duration: 6 years, 25 years, and lifetime). A second assumption is that the individual's preference for a medical intervention is determined by the levels of those attributes [13]. The relative importance of attributes and the trade-offs that respondents make between them can be assessed by offering a series of choices between two or more medical intervention alternatives with different combinations of attribute levels (see Table 1 for an example of a choice set) [14]. In comparison to other stated preference techniques, a DCE presents a reasonably straightforward task and one which more closely resembles a real-world decision, i.e. trading-off health and non-health outcomes [15]. 


\subsection{Attributes and attribute levels}

We selected the most relevant HPV vaccination attributes and their levels based on the literature, interviews with experts in the field of HPV vaccination $(n=8)$, and focus groups data ( $\mathrm{n}=4 ; 36$ parents participated ( 34 female and 2 male), aged 33 to 53 years with at least one child in the age of 8 to 14 years. We did not include girls in the focus groups, because at that time it was expected that the target group for HPV vaccination would be 9-year old girls, who are under Dutch law not allowed to decide themselves about the vaccination uptake). In the focus groups we collected data on the attributes that individuals expected to be important or that had been important in their decision to participate in an HPV vaccination programme. Experts were asked to comment on a list of attributes, which were derived from a literature review, and to rank them in order of importance. Based on these data we selected the five most important attributes as identified by both groups: 1) degree of protection against cervical cancer; 2) protection duration; 3) serious side-effects (e.g. hospitalization); 4) mild sideeffects (e.g. nausea); and 5) age of vaccination. Attributes that were plausible and relevant both clinically and from a policy viewpoint were determined. A sufficiently wide range of levels was used to avoid respondents ignoring attributes because of too small differences in levels. The attributes and levels are presented in Table 2.

\subsection{Study design and questionnaire}

The combination of five attributes with three levels each resulted in $243\left(3^{5}\right)$ hypothetical HPV vaccination alternatives. Since it is not feasible to present a single individual with all these alternatives (i.e. full factorial design), we generated a sample of alternatives from all these 243 alternatives (i.e., we used a fractional factorial design) by means of a catalogue, which contains a library of orthogonal arrays [16]. Fifty-four HPV vaccination alternatives 
proved sufficient to estimate all main effects and a number of two-way interactions between attributes in a regression analysis. In this fractional factorial design, attributes were independent of each other, thus guaranteeing orthogonality (i.e. the design was defined in such a way that the attributes could not represent the same facts), and attribute levels occurred with equal frequency, maintaining level balance [17]. Choice sets were designed using the discrete choice experiment software of Street and Burgess [18]. Our design, which contained 54 choice sets, had an efficiency of $82 \%$ compared with an optimal choice design. This means that our design was a near optimal design that counterbalanced statistical reasons and practical reasons (a higher amount of choice sets will result in a more precise estimation of the coefficients, however as a consequence (much) more respondents are needed). Choice sets consisted of two HPV vaccination alternatives and a 'no HPV vaccination' option to allow respondents to 'opt out' (Table 1); HPV vaccination is a preventive medical intervention and, as in real life, respondents are not obliged to opt for HPV vaccination. Respondents were asked to consider all three options in a choice set as realistic alternatives and to choose the option that appealed most to them. Presenting a single individual with a large amount of choice sets is expected to result in a lower response rate and/or lower response reliability [19, 20]. To avoid this, we used a blocked design [14], which resulted in dividing the 54 choice sets over six types of questionnaires containing nine choice sets each.

Each questionnaire started with a detailed description of the attributes and their levels (the (complete) questionnaire is available from the authors on request). Pictures, graphs and pictograms were included to demonstrate percentages and rates. To assess the understanding of the attributes (protection levels against cervical cancer, levels of serious side effects, and levels of mild side-effects) the questionnaire contained a dominant choice set (rationality test). In this set one of two HPV vaccination alternatives was characterised by equal or logically preferable levels on all attributes. 
The main part of each questionnaire comprised nine choice sets. Furthermore, the following data were collected: age at completing the questionnaire, level of education, religious affiliation, country of birth, parents' countries of birth, history of childhood vaccinations, and of vaccinations against HPV. To check the convergent validity of the DCE, respondents were asked to rank the five attributes of HPV vaccination from most important to least important. The questionnaire was pilot tested to check for any problems in interpretation and face validity $(n=16)$.

\subsection{Study sample}

A representative sample of 359 girls aged 12 to 16 years were randomly approached at 30 classes from four secondary schools and cities located in the north-east (rural area) and west part (urban area) of the Netherlands. This age range was chosen based on current Dutch policy guideline. Calculation of optimal sample sizes for estimating non-linear discrete choice models from DCE data is complicated as it depends on the true values of the unknown parameters estimated in the choice models [21]. Lancsar and Louviere [21] mentioned that one rarely requires more than 20 respondents per parameter to estimate reliable models; our DCE contained eight parameters in the main effects model (see Equation 1), which meant that we had to include at least 160 respondents. Taking into account a suboptimal response rate, and some two-way interactions between attributes, we aimed at having at least 300 questionnaires completed.

\subsection{Procedure}

Questionnaires were completed in the classroom or auditorium in the presence of a researcher or assistant. First, general information was given about HPV, cervical cancer, the causal link between them, HPV vaccinations, cervical cancer screening and the NIP ( \pm 5 minutes). This 
was followed by an explanation of DCE questions ( \pm 5 minutes). Subsequently, respondents completed the questionnaire on paper ( \pm 20 -30 minutes). The whole procedure lasted at most 45 minutes. Beforehand parents had received an information letter covering the purpose, voluntariness and anonymity of the study and an opt-out form. Approval for the study was obtained from the Medical Ethics Committee, Erasmus MC, University Medical Centre Rotterdam.

\subsection{Statistical analyses}

The DCE was analysed by taking each choice among the three options (two HPV vaccination alternatives, and a 'no HPV vaccination' alternative) as an observation, i.e. two 'no' and one 'yes'. The observations were analysed by a mixed logit regression model to take heterogeneity as well as correlation between the choice task completed by each individual into account [14]. After testing for linear continuous effects of one or more attributes, the following utility model was estimated:

$$
\begin{aligned}
& \mathrm{V}=\beta_{0}+\beta_{1} \text { EFFECTIVENESS }+\beta_{2} \text { DURATION_25Y }+\beta_{3} \text { DURATION_LIFETIME }+ \\
& \beta_{4} \text { SERIOUS_1/150,000 }+\beta_{5} \text { SERIOUS_1/30,000 }+\beta_{6} \text { MILD }+\beta_{7} \text { AGE_12Y }+\beta_{8} \text { AGE_14Y }
\end{aligned}
$$

$\mathrm{V}$ is the observable relative utility that is composed of the preference scores for the individual $\beta$-coefficients of the model. $\beta_{0}$ is a constant reflecting respondents' preference for receiving HPV vaccination relative to 'no HPV vaccination'. $\beta_{1}-\beta_{8}$ are coefficients of the attributes indicating the relative weight individuals place on a certain attribute(level). The statistical significance of a coefficient ( $\mathrm{p}$-value $\leq 0.05$ ) indicates that individuals differentiated between one attribute (or attribute level) and another in making stated choices. A priori, we expected 
all attributes to be statistically significant. The sign of a coefficient reflects whether the attribute has a positive or negative effect on preference score. We expected that only the attribute 'mild' and the estimated attribute levels of 'serious side-effects' would have a negative effect (i.e., a negative sign).

The value of each coefficient represents the importance respondents assign to an attribute(level). However, different attributes utilise different units of measurement. For example, the coefficient for 'protection against cervical cancer' represents the importance per absolute $10 \%$ protection rate. When looking at an HPV vaccination that generates a $70 \%$ protection rate, the coefficient should be multiplied seven times $(7 *$ coefficient of 'protection against cervical cancer' of $10 \%=$ coefficient of 'protection against cervical cancer' of $70 \%$ ). To explore the impact of respondents who failed the rationality test, sensitivity analyses were conducted by excluding such individuals from the sample and rerunning the analysis.[22, 23] Also, two-way interactions were added to the main effects model to test which two-way interactions were significant and improved the fit of the model.

To investigate the willingness of girls to trade-off protection against cervical cancer to achieve an improvement in one level of the other HPV vaccination attributes, we calculated the ratios between the coefficients of the attributes with protection against cervical cancer as the denominator. For example, $-\beta_{6} / \beta_{1}$ indicates how much protection against cervical cancer girls were willing to forego to get an HPV vaccination programme that had a five percent lower risk in mild side-effects.

Finally, choice probabilities were also calculated to provide a way to convey DCE results to decision makers that is more easily understandable. The probability that an individual says "yes" to an HPV vaccination programme is equal to:

$$
\mathrm{P}=1 /\left(1+\mathrm{e}^{-\mathrm{V}}\right)
$$


where $\mathrm{V}$ is defined as in Equation 1. We calculated the choice probability (i.e. the mean uptake) for the base case. The base case used in this study represents an HPV vaccination programme at the age of 12 years, a 1/30 risk of mild side-effects, a 1/150,000 risk of serious side-effects; a protection duration of 6 years, and a $70 \%$ protection against cervical cancer. We presented these results in a 'tornado' graph [17] to illustrate the marginal effect of varying one attribute level at a time from the base case, holding all other attributes constant. This base was chosen to correspond i) with an HPV vaccination programme that contained most plausible levels based on literature, and ii) with the Dutch situation (HPV vaccination programme at the age of 12 years). The graph shows how each attribute systematically affects choices relative to the base case. Noteworthy, in the calculation of the mean uptake we took all heterogeneity into account as the mean uptake is not just equal to the uptake of someone with average coefficient values. Additionally, we calculated the minimum acceptable efficacy and maximum acceptable risk of mild side-effects, in which the base case HPV vaccination programme is preferred over no HPV vaccination (i.e. relative utility composed of the preference scores for the individual $\beta$-coefficients and standard deviations of the model is higher than zero). 


\section{Results}

\subsection{Respondents}

The response rate was $312 / 359(87 \%)$. The respondents had a mean age of 13.3 years $(\mathrm{SD}=1.0)$. Of all respondents, $58 \%$ had at least one dose of HPV-vaccine, $62 \%$ had a higher secondary educational level, and 38\% considered themselves to be religious (Table 3). Results of direct ranking showed that the protection against cervical cancer, the protection duration, and the risk of serious side-effects of HPV vaccination were considered the most important attributes of an HPV vaccination programme (Figure 1).

\subsection{DCE results}

The 'no HPV vaccination' option was chosen in $21.4 \%$ of the choice sets. Twenty-one out of 312 girls $(6.7 \%)$ always chose the 'no HPV vaccination' option. All five vaccination characteristics proved to influence girls' preferences for HPV vaccination ( $p<0.05$; Table 4). The positive or negative directions of the coefficients of the characteristics were consistent with our a priori hypotheses and showed, therefore, theoretical validity. The positive sign given to the coefficients 'degree of protection against cervical cancer' and 'protection duration' indicated that respondents preferred an HPV vaccination generating a higher degree of protection and a longer protection duration over an HPV vaccination that generates a lower degree of protection and a shorter protection duration. The negative signs for 'side-effects' indicate that girls preferred an HPV vaccination programme with low serious and low mild side-effects. The non-significant coefficient of the characteristic level 'vaccination at age 14 years' indicated that respondents did not significantly prefer this age of vaccination over a vaccination at age 9 years. However, respondents significantly preferred vaccination at age 12 years over vaccination at age 9 years. Most estimated standard deviations were significant, 
which indicated preference heterogeneity among girls for several characteristics of HPV vaccination.

The results of the sensitivity analyses indicated that i) excluding respondents who 'failed' the rationality test $(2.6 \%$ of the respondents) had no relevant impact on the size or relative importance of the attributes, and ii) none of the two-way interactions were significant and improved the fit of the model (data not shown).

Comparing our DCE results with the results of the direct ranking in our questionnaire, both preference methods showed that protection against cervical cancer, protection duration, and risk of serious side-effects of HPV vaccination were considered the most important attributes of an HPV vaccination programme. These results support convergent validity of the DCE results.

\subsection{Trade-offs}

Based on the expressed preferences, girls showed their willingness to trade-off protection against cervical cancer to achieve an improvement in one level of the other HPV vaccination attributes (Table 5). On average, girls were willing to trade-off $38 \%$ protection against cervical cancer to obtain an HPV vaccination programme with a life-time protection duration instead of a protection duration of 6 years. Girls were willing to trade-off $17 \%$ protection against cervical cancer to obtain a vaccination with a risk of serious side effects of $1 / 750,000$ instead of $1 / 150,000,9 \%$ protection against cervical cancer to get an HPV vaccination that had a five percent lower risk in mild side-effects, and $7 \%$ protection against cervical cancer to get an HPV vaccination at age 12 years instead of age 9 years. Considering the relative tradeoff between the risk of mild and serious side effects, girls were willing to accept a $9.7 \%(7.1 \%$ to $13.2 \%$ ) increased risk of mild side effects if the risk of serious side effects decreased from $1 / 150,000$ to $1 / 750,000$. 


\subsection{Expected uptake of HPV-vaccination}

We found an expected uptake of the base case HPV vaccination programme (70\% protection against cervical cancer, at age 12 years, $1 / 30$ risk of mild side-effects, $1 / 150,000$ risk of serious side-effects, and protection duration of 6 years) of approximately $77 \%$ (CI: $74-80 \%$ ). Especially an increased risk of serious side-effects from $1 / 150,000$ to $1 / 30,000$, a life-time protection instead of a protection duration of 6 years, or a decrease in protection against cervical cancer from $70 \%$ to $50 \%$ had a relatively large impact on the average expected uptake (a decrease of $14.6 \%$, an increase of $12.0 \%$, and a decrease of $7.3 \%$, respectively) (Figure 2). Assuming an HPV vaccination at age 12 years, a 1/30 risk of mild side-effects, a $1 / 150,000$ risk of serious side-effects, and a protection duration of 6 years, the minimum efficiency of this HPV vaccination should be $15 \%$ to be preferred over no vaccination. Or assuming an HPV vaccination at age 12 years, a 1/150,000 risk of serious side-effects, a protection duration of 6 years, and a $70 \%$ protection rate against cervical cancer, the maximum risk for mild side effects should be $34 \%$ to be preferred over no vaccination. 


\section{Discussion}

The present study shows that girls made a trade-off between vaccine characteristics. Degree of protection against cervical cancer, duration of protection, risk of serious side-effects (e.g. hospitalization), risk of mild side-effects (e.g. nausea), and age of vaccination, all proved to influence girls' preferences for HPV vaccination. On average, girls were willing to forego protection against cervical cancer if the protection duration of HPV vaccination was longer, or if the risk of serious or mild side-effects of HPV vaccination was lower. An increase in protection duration, an increase in risk of serious side-effects, or a decrease in degree of protection against cervical cancer had a relatively large impact on the average expected uptake.

There are no previous DCEs investigating how characteristics of HPV vaccination determine girls' preferences for participation in HPV vaccination. However, Dahlström et al. [24] investigated the attitudes to HPV vaccination among parents of children aged 12-15 years. They found that beliefs about vaccine safety and efficacy were strong correlates of willingness to vaccinate. Dempsey et al. [25], who investigated the reasons why mothers do or do not have their adolescent daughters vaccinated against HPV, concluded that addressing safety concerns may be one of the most useful targets for future interventions to increase HPV vaccine utilisation. Brown et al. [26], who estimated how features of HPV vaccines affect mothers' perceived benefit for daughters aged 13-17 years, showed that cervical cancer protection and duration of effectiveness were the most important attributes. All these results are in line with the findings of our study, which show that protection against cervical cancer, protection duration, and serious side-effects play an important role in girls' choices for HPV vaccination. In a vaccination context, Hall et al. [27] used a DCE to study the introduction of varicella vaccination. They showed that immunisation rates would increase in case of a lower incidence of mild and severe side-effects, which is similar to our study results. 
The possibility to estimate the willingness to forego protection against cervical cancer is an additional advantage of DCE. However, in our opinion this additional advantage is limited. In the context of willingness to pay (WTP), earlier studies showed that the WTP derived from a DCE changed if a wider cost range was chosen [28], or that the WTP derived from an openended question differed from the WTP derived from a DCE [29]. This same phenomenon might be possible for the willingness to forego protection against cervical cancer derived from a DCE. Further research in this area is needed and, meanwhile, we recommend the interpretation of these absolute willingness values to forego protection against cervical cancer in a relative manner (i.e. ranking order).

Our results showed that the expected uptake of the base case HPV vaccination programme was much higher $(76 \%)$ than the attendance rate in the first HPV vaccination round in the Netherlands in 2009 (49\%) [30]. This 49\% is also relatively low compared to the Dutch National Immunisation Programme for protection against childhood infectious disease (>95\%).[31] Possible clarifications are uncertainty about the degree of protection against cervical cancer, protection duration, and serious side-effects (all of which played the most important role in girls' preferences for HPV vaccination). To date, follow-up data on HPV vaccinated young women are available for 7.3 years $[32,33]$.

The present study had several limitations. First, our sample contained a relatively large number of high educated respondents, which precludes generalisation of the findings to all girls. Second, we selected the most relevant attributes in our DCE using literature, interviews with experts in the field of HPV vaccination, and focus group data; however, this careful procedure does not guarantee that we included all attributes that are relevant to girls' preferences for HPV vaccination. Third, we did not include genital warts protection as an attribute of HPV vaccination as we did not receive signals that genital warts protection would play a role in the decision about HPV vaccination uptake, and as the Dutch vaccination 
programme offers only Cervarix, which provides no protection against HPV types causing warts. However, girls may well have a preference for HPV vaccines offering warts protection. Fourth, the inclusion of percentages and rates in our discrete choice experiment, especially the inclusions of small risk levels, might have caused difficulties with understanding the choice task. Finally, the current results should preferably be validated by comparing them with the actual behaviour of girls in an HPV vaccination programme.

In conclusion, this study shows that girls made trade-offs between protection against cervical cancer and other characteristics of HPV vaccination. Especially the degree of protection against cervical cancer, protection duration, and risk of serious side-effects influenced HPV vaccination preferences. We conclude that, uptake of HPV vaccination may change considerably if girls are supplied with new evidence-based information about the degree of protection against cervical cancer, the protection duration, and the risk of serious side-effects. 


\section{Acknowledgement}

The authors thank the two anonym researchers for their valuable comments and suggestions, and the Dutch Cancer Society (no. EMCR 2008-3992) for their grant support. 


\section{References}

[1] Bray F, Loos AH, McCarron P, Weiderpass E, Arbyn M, Moller H, et al. Trends in cervical squamous cell carcinoma incidence in 13 European countries: changing risk and the effects of screening. Cancer Epidemiol Biomarkers Prev 2005 Mar;14(3):677-86.

[2] Walboomers JM, Jacobs MV, Manos MM, Bosch FX, Kummer JA, Shah KV, et al. Human papillomavirus is a necessary cause of invasive cervical cancer worldwide. J Pathol 1999 Sep;189(1):12-9.

[3] Schiffman M, Castle PE, Jeronimo J, Rodriguez AC, Wacholder S. Human papillomavirus and cervical cancer. Lancet 2007 Sep 8;370(9590):890-907.

[4] Clifford G, Franceschi S, Diaz M, Munoz N, Villa LL. Chapter 3: HPV typedistribution in women with and without cervical neoplastic diseases. Vaccine 2006 Aug 31;24 Suppl 3:S3/26-34.

[5] De Vuyst H, Clifford G, Li N, Franceschi S. HPV infection in Europe. Eur J Cancer 2009 Oct;45(15):2632-9.

[6] Levy-Bruhl D, Bousquet V, King LA, O'Flanagan D, Bacci S, Lopalco PL, et al. The current state of introduction of HPV vaccination into national immunisation schedules in Europe: results of the VENICE 2008 survey. Eur J Cancer 2009 Oct;45(15):2709-13.

[7] Phillips KA, Van Bebber S, Marshall D, Walsh J, Thabane L. A review of studies examining stated preferences for cancer screening. Prev Chronic Dis 2006 Jul;3(3):A75.

[8] Conroy K, Rosenthal SL, Zimet GD, Jin Y, Bernstein DI, Glynn S, et al. Human papillomavirus vaccine uptake, predictors of vaccination, and self-reported barriers to vaccination. Journal of women's health (2002) 2009 Oct;18(10):1679-86.

[9] Kahn JA, Rosenthal SL, Jin Y, Huang B, Namakydoust A, Zimet GD. Rates of human papillomavirus vaccination, attitudes about vaccination, and human papillomavirus prevalence in young women. Obstetrics and gynecology 2008 May;111(5):1103-10. 
[10] Mathur MB, Mathur VS, Reichling DB. Participation in the decision to become vaccinated against human papillomavirus by California high school girls and the predictors of vaccine status. J Pediatr Health Care Jan-Feb;24(1):14-24.

[11] Ryan M, Gerard K. Using discrete choice experiments to value health care programmes: current practice and future research reflections. Appl Health Econ Health Policy 2003;2(1):55-64.

[12] De Bekker-Grob EW, Ryan M, Gerard K, Essink-Bot ML, Steyerberg EW. Applying discrete choice experiments to value health and health care: a review of the literature. Submitted.

[13] Ryan M. Discrete choice experiments in health care. Bmj 2004 Feb 14;328(7436):3601.

[14] Hensher DA, Rose JM, Greene WH. Applied choice analysis: a primer. Cambridge: Cambridge University Press, 2005.

[15] Mangham LJ, Hanson K, McPake B. How to do (or not to do) ... Designing a discrete choice experiment for application in a low-income country. Health Policy Plan 2009 Mar;24(2):151-8.

[16] Hahn GJ, Shapiro SS. A catalogue and computer program for the design and analysis of orthogonal symmetric and asymmetric fractional factorial experiments. Schenectady, NY, USA: General Electric Research and Development Centre; 1966.

[17] Louviere JJ, Hensher DA, Swait JD. Stated choice methods: analysis and application. Cambridge: Cambridge University Press, 2000.

[18] Street D, Burgess L. Discrete choice experiments [computer software]. 2007 [cited; Available from: http://maths.science.uts.edu.au/maths/wiki/SPExpts [Accessed 2009 Oct 26] 
[19] Hall J, Fiebig DG, King MT, Hossain I, Louviere JJ. What influences participation in genetic carrier testing? Results from a discrete choice experiment. J Health Econ 2006 May;25(3):520-37.

[20] Pearmain D, Swanson J, Kroes E, Bradley M. Stated preferences techniques: a guide to practice. The Hague, Steer Davis Gleave and Hague Consulting Group. 1991.

[21] Lancsar E, Louviere J. Conducting discrete choice experiments to inform healthcare decision making: a user's guide. Pharmacoeconomics 2008;26(8):661-77.

[22] Lancsar E, Louviere J. Deleting 'irrational' responses from discrete choice experiments: a case of investigating or imposing preferences? Health Econ 2006 Aug;15(8):797-811.

[23] Ryan M, Watson V, Entwistle V. Rationalising the 'irrational': a think aloud study of discrete choice experiment responses. Health Econ 2009 Mar;18(3):321-36.

[24] Dahlstrom LA, Tran TN, Lundholm C, Young C, Sundstrom K, Sparen P. Attitudes to HPV vaccination among parents of children aged 12-15 years-a population-based survey in Sweden. Int J Cancer Jan 15;126(2):500-7.

[25] Dempsey AF, Abraham LM, Dalton V, Ruffin M. Understanding the reasons why mothers do or do not have their adolescent daughters vaccinated against human papillomavirus. Ann Epidemiol 2009 Aug;19(8):531-8.

[26] Brown DS, Johnson FR, Poulos C, Messonnier ML. Mothers' preferences and willingness to pay for vaccinating daughters against human papillomavirus. Vaccine 2009 Dec 28.

[27] Hall J, Kenny P, King M, Louviere J, Viney R, Yeoh A. Using stated preference discrete choice modelling to evaluate the introduction of varicella vaccination. Health Econ 2002 Jul;11(5):457-65. 
[28] Slothuus Skjoldborg U, Gyrd-Hansen D. Conjoint analysis. The cost variable: an Achilles' heel? Health Econ 2003 Jun;12(6):479-91.

[29] Marjon van der P, Shiell A, Au F, Johnston D, Tough S. Convergent validity between a discrete choice experiment and a direct, open-ended method: comparison of preferred attribute levels and willingness to pay estimates. Soc Sci Med 2008 Dec;67(12):2043-50.

[30] National Institute for Public Health and the Environment. HPV nieuws; 17 december 2009;

$\mathrm{nr}$

(http://www.rivm.nl/cib/binaries/09\%20HPV\%20nieuws\%20interactief\%2021_tcm92-

65485.pdf). 2009 [cited; Available from:

http://www.rivm.nl/cib/binaries/09\%20HPV\%20nieuws\%20interactief\%2021_tcm92-

65485.pdf

[31] Nederlands Vaccin Instituut. RVP (National Immunization Program) (http://www.nvivaccin.nl/?id=344). [cited; Available from: http://www.nvi-vaccin.nl/?id=344

[32] GlaxoSmithKline Vaccine HPVSG, Romanowski B, de Borba PC, Naud PS, RoteliMartins CM, De Carvalho NS, et al. Sustained efficacy and immunogenicity of the human papillomavirus (HPV)-16/18 AS04-adjuvanted vaccine: analysis of a randomised placebocontrolled trial up to 6.4 years. Lancet 2009 Dec 12;374(9706):1975-85.

[33] De Carvalh N, Roteli-Martin C, Teixeira J, Naud P, de Borba P, Zahaf T, et al. Immunogenicity and safety of HPV-16/18 AS04-adjuvanted vaccine up to 7.3 years. Abstract presented at the 25th IPV conference. 8- 14 May 2009; Malmo, Sweden. 2009. 
Table 1: Example of choice set

\begin{tabular}{|l|c|c|c|}
\hline Attributes & Program A & Program B & No vaccination \\
\hline $\begin{array}{l}\text { Protection against cervical } \\
\text { cancer }\end{array}$ & $70 \%$ & $90 \%$ & $0 \%$ \\
\hline Protection duration & Lifetime & 6 years & n.a. \\
\hline Serious side-effects & $1: 750,000$ & $1: 750,000$ & No risk \\
\hline Mild side-effects & $1: 50$ & $1: 30$ & No risk \\
\hline Age at vaccination & 14 years & 9 years & n.a. \\
\hline $\begin{array}{l}\text { Which vaccination } \\
\text { program do you prefer? }\end{array}$ & $\square \mathbf{A}$ & $\square \mathbf{B}$ & $\square$ None \\
\hline
\end{tabular}

n.a. = not applicable 
Table 2: Considered attributes and attribute levels for HPV vaccination

\begin{tabular}{lll}
\hline & \multicolumn{2}{c}{ Regression analysis } \\
\cline { 2 - 3 } Attributes and levels & Coefficient & Attribute name \\
\hline Protection against cervical cancer & $\beta_{1}$ & EFFECTIVENESS \\
$50 \%$ & & \\
$70 \%$ & & \\
$90 \%$ & & \\
Protection duration & $\beta_{2}$ & DURATION_25Y \\
6 years (reference level) & $\beta_{3}$ & DURATION_LIFETIME \\
25 years & & \\
lifetime & & \\
Serious side effects & $\beta_{4}$ & SERIOUS_1/150,000 \\
$1 / 750,000$ & $\beta_{5}$ & SERIOUS_1/30,000 \\
$1 / 150,00$ & $\beta_{6}$ & MILD \\
$1 / 30,000$ & & \\
Mild side effects & & \\
$1 / 50$ & & AGE_12Y \\
$1 / 30$ & & AGE_14Y \\
$1 / 10$ & $\beta_{7}$ & $\beta_{8}$
\end{tabular}


Table 3: Respondent characteristics

Characteristics

\begin{tabular}{lrr} 
& (n=312) \\
\hline & Mean & (SD) \\
Age (years) & 13.3 & $(1.0)$ \\
& $\mathbf{n}$ & $(\%)$ \\
Educational level & & \\
$\quad$ Lower secondary education & 38 & $(12.2)$ \\
Intermediate secondary education & 81 & $(26.0)$ \\
Higher secondary education & 193 & $(61.9)$ \\
Religion & & \\
$\quad$ None & 191 & $(61.2)$ \\
Christian (incl. Catholic, Protestant) & 104 & $(33.3)$ \\
Moslim & 11 & $(3.5)$ \\
Other & 4 & $(1.3)$ \\
Country of birth & & \\
The Netherlands & 293 & $(93.9)$ \\
Other (UK, France, Poland, Albania, Mexico, Aruba, & 15 & $(4.9)$ \\
Afghanistan, Pakistan, China, India, Iraq, Kazakhstan, & & \\
Philippines & & \\
Country of birth of parents & & \\
Both parents in the Netherlands & & \\
One parent outside the Netherlands & 256 & $(82.1)$ \\
Both parents outside the Netherlands & 23 & $(7.4)$ \\
HPV vaccinated & 26 & $(8.3)$ \\
Yes & & \\
Vaccinated against childhood diseases & 181 & $(58.0)$ \\
$\quad$ Yes & & \\
$\quad$ No & 259 & $(83.0)$ \\
Unknown & 5 & $(1.6)$ \\
\hline & & $(15.1)$ \\
\hline
\end{tabular}


Table 4: Girls' preferences for HPV vaccination

\begin{tabular}{|c|c|c|c|}
\hline \multirow[t]{2}{*}{ Attributes } & \multirow[t]{2}{*}{ Coefficient } & \multicolumn{2}{|l|}{ Mixed logit } \\
\hline & & Value & $(95 \% \mathrm{CI})$ \\
\hline \multirow[t]{2}{*}{$\overline{\text { Constant (vaccination) }}$} & Mean & -0.28 & $(-0.92$ to 0.36$)$ \\
\hline & S.D. & $3.60 * * *$ & (3.03 to 4.17$)$ \\
\hline \multirow[t]{2}{*}{ Protection against cervical cancer (per 10\%) } & Mean & $0.64 * * *$ & $(0.55$ to 0.72$)$ \\
\hline & S.D. & $0.36 * * *$ & ( 0.30 to 0.42$)$ \\
\hline \multirow[t]{2}{*}{ Protection duration 6 years (omitted) } & Mean & $-1.41 * * *$ & $(-1.70$ to -1.12$)$ \\
\hline & S.D. & $0.88 * * *$ & $(0.87$ to 0.90$)$ \\
\hline \multirow[t]{2}{*}{ Protection duration 25 years } & Mean & $0.20 * * *$ & (0.08 to 0.33$)$ \\
\hline & S.D. & 0.07 & $(-0.22$ to 0.35$)$ \\
\hline \multirow[t]{2}{*}{ Protection duration lifetime } & Mean & $1.20 * * *$ & (1.03 to 1.37$)$ \\
\hline & S.D. & $0.88 * * *$ & (0.71 to 1.05$)$ \\
\hline \multirow[t]{2}{*}{$1 / 750,000$ risk on serious side effects (omitted) } & Mean & $2.15 * * *$ & (1.89 to 2.40$)$ \\
\hline & S.D. & $0.80 * * *$ & $(0.78$ to 0.83$)$ \\
\hline \multirow[t]{2}{*}{$1 / 150,000$ risk on serious side effects } & Mean & $-0.55 * * *$ & $(-0.68$ to -0.43$)$ \\
\hline & S.D. & $0.18 *$ & $(0.04$ to 0.40$)$ \\
\hline \multirow[t]{2}{*}{$1 / 30,000$ risk on serious side effects } & Mean & $-1.60 * * *$ & $(-1.78$ to -1.42$)$ \\
\hline & S.D. & $0.78 * * *$ & (0.56 to 1.01$)$ \\
\hline \multirow[t]{2}{*}{ Mild side effects (per 5\%) } & Mean & $-0.57 * * *$ & $(-0.71$ to -0.44$)$ \\
\hline & S.D. & $0.50 * * *$ & $(0.30$ to 0.71$)$ \\
\hline \multirow[t]{2}{*}{ Vaccination at age 9 years (omitted) } & Mean & $-0.24 * * *$ & $(-0.37$ to -0.11$)$ \\
\hline & S.D. & $0.34 * * *$ & (0.32 to 0.35$)$ \\
\hline \multirow[t]{2}{*}{ Vaccination at age 12 years } & Mean & $0.21 * * *$ & (0.09 to 0.33$)$ \\
\hline & S.D. & 0.04 & $(-0.36$ to 0.27$)$ \\
\hline \multirow[t]{2}{*}{ Vaccination at age 14 years } & Mean & 0.03 & $(-0.08$ to 0.14$)$ \\
\hline & S.D. & $0.34 * * *$ & $(0.18$ to 0.49$)$ \\
\hline Number of responses & \multicolumn{3}{|c|}{8,424} \\
\hline Number of respondents & \multicolumn{3}{|c|}{312} \\
\hline Log-likelihood & \multicolumn{3}{|c|}{$-1,735.60$} \\
\hline
\end{tabular}

Notes: (1) Effects coded variables used for protection duration, serious side effects, and age at vaccination; (2) Normal distribution for random coefficients used on all attributes; (3) The value of the omitted term equals the negative sum of the coefficients of the included attributes; (4) *** denotes $\mathrm{p}<.01$, ** $\mathrm{p}<.05$, $* \mathrm{p}<0.10$ for statistical significance; (5) S.D. = standard deviation 
Table 5: Girls' trade-offs between risk reduction and different aspects of a vaccination programme

\begin{tabular}{lccl}
\hline & Girls & Interpretation note \\
\hline & $\begin{array}{c}\text { were willing to forego } \\
\text { protection against cervical } \\
\text { cancer of...(\%; CI) }\end{array}$ \\
\hline Protection duration & 37.8 & $(32.1$ to 44.3$)$ & ....to get a vaccination with life-time protection instead of a protection duration of 6 years \\
Serious side effects & 17.4 & $(13.4$ to 22.0$)$ & ....to get a vaccination with a risk of serious side effects of $1 / 750,000$ instead of $1 / 150,000$ \\
Mild side effects & 9.0 & $(6.9$ to 11.2$)$ & ....to get a vaccination with a $5 \%$ lower risk of mild side-effects \\
Age of vaccination & 6.6 & $(2.6$ to 10.6$)$ & ...to get a vaccination at age 12 years instead of age 9 years \\
\hline
\end{tabular}


Figure 1: Most important vaccination characteristic based on direct ranking ( $\mathrm{n}=290$ respondents).

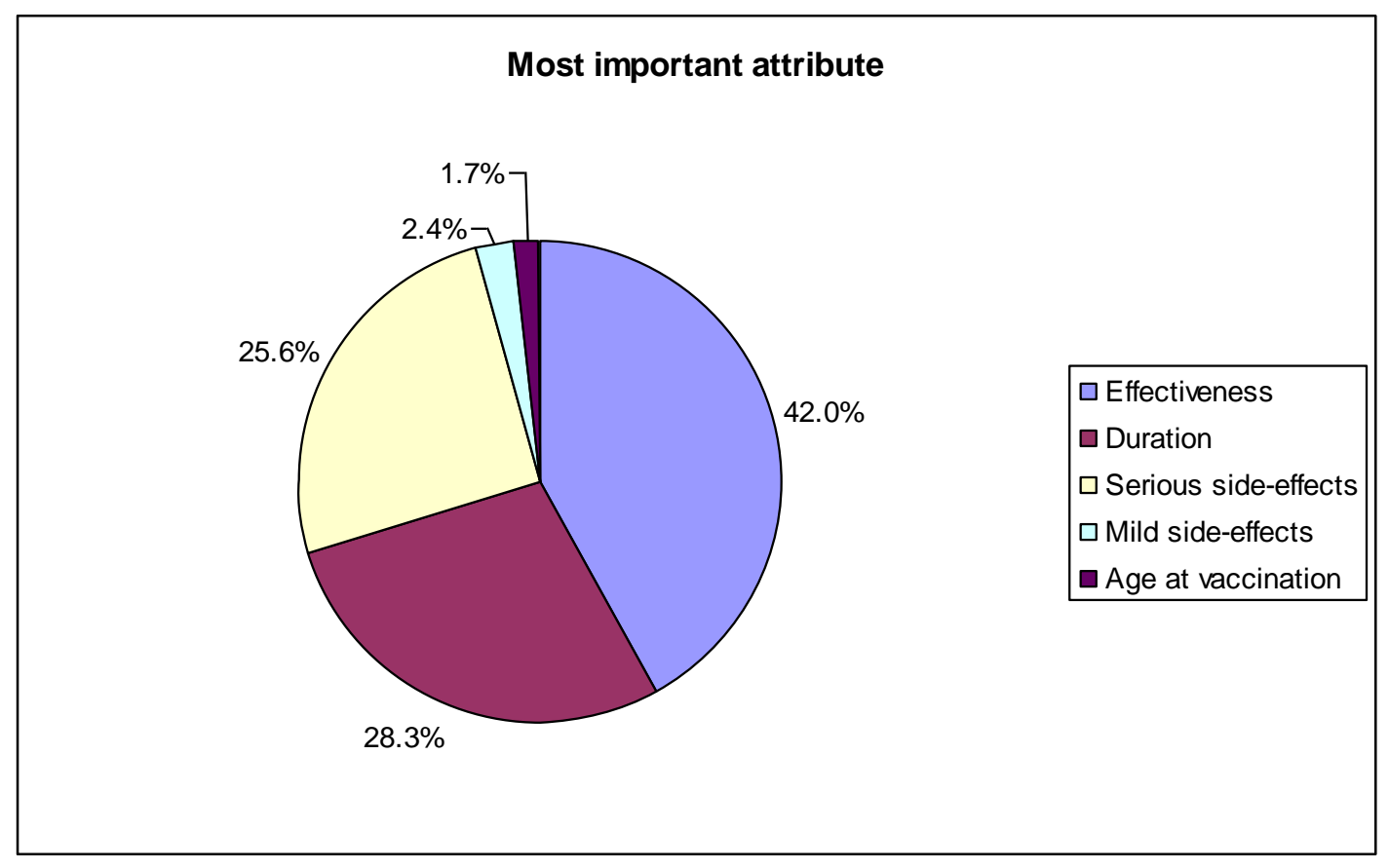


Figure 2: Univariate marginal estimates for predicted probability of participation; highest and lowest values for attributes level changes versus base case

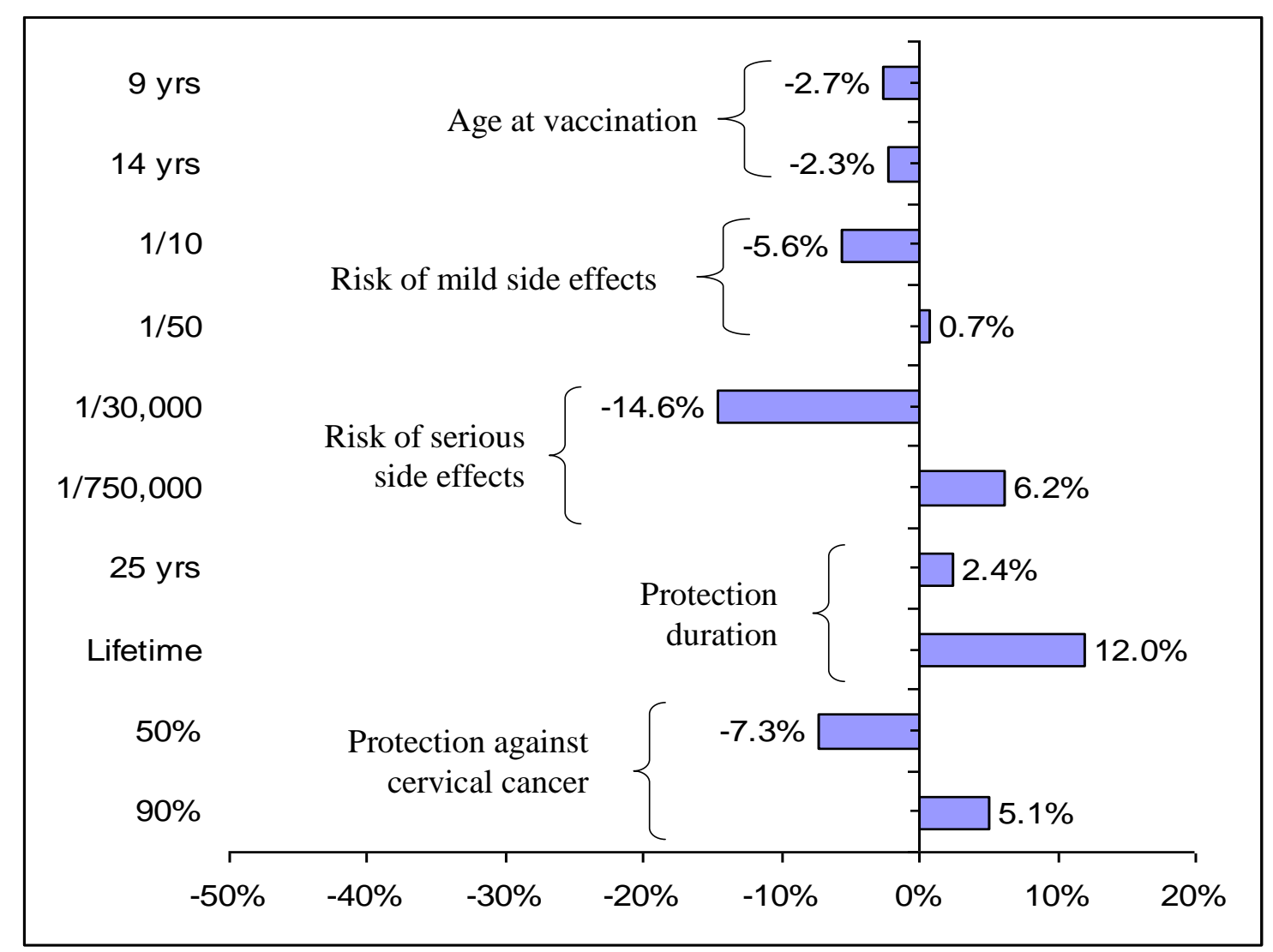

Note: The base case is an HPV vaccination at age 12 years, 1/30 risk of mild side-effects, 1/150,000 risk of serious side-effects; protection duration of 6 years and $70 \%$ protection against cervical cancer. This base case is indicated as zero change in the probability of the $\mathrm{x}$ axis. 